\title{
Prescrição de exercício físico: a sua inclusão na consulta
}

Diana Carneiro*

\section{RESUMO}

A percentagem de indivíduos com hábitos sedentários tem aumentado nos últimos anos, sendo o sedentarismo um factor de risco importante das doenças crónicas não transmissíveis, nomeadamente das doenças cardiovasculares. Assim, é de extrema importância que ocorram alterações na nossa sociedade, nomeadamente, nas atitudes, nos valores e na conduta no que concerne à relação entre a saúde e o exercício físico.

Há evidência que a inclusão do exercício físico na prescrição médica é um caminho viável para diminuir os níveis de sedentarismo da população em geral. Os médicos de família encontram-se numa posição privilegiada para tentar mudar o comportamento das pessoas, não só pelo acompanhamento longitudinal e continuado da população, mas também pelo conhecimento do meio familiar, ambiental e comunitário que rodeia os indivíduos.

A prescrição de exercício físico deve ser vista como um processo pelo qual se recomenda um programa de exercícios de forma sistemática e individualizada, segundo as necessidades e preferências de cada pessoa, com a finalidade de obter os maiores benefícios com os menores riscos. Não basta indicar que se deve fazer exercício. À semelhança de como se faz com qualquer outra medicação, é necessário especificar os aspectos quantitativos e qualitativos do exercício físico.

Com este artigo pretende-se chamar a atenção para a prescrição de exercício físico e abordá-lo como um dos actos médicos que pode ser realizado em consulta. Faz-se referência aos benefícios do exercício físico, ao papel do médico como prescritor de exercício físico, aos modelos e teorias de comportamento que visam a prática de exercício físico, às características da prescrição de exercício físico, às estratégias a implementar na prática clínica e aos programas baseados no aconselhamento de exercício físico desenvolvidos nos cuidados de saúde primários.

Perante um aumento significativo dos hábitos sedentários da nossa população, torna-se urgente rever o aconselhamento de exercício físico nos cuidados de saúde primários.

Palavras-chave: Estilo de Vida Sedentário; Mudança de Comportamentos; Exercício; Aconselhamento.

\section{INTRODUÇÃO}

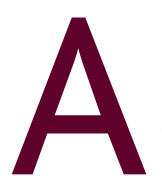
sociedade actual tem vindo a assistir ao aumento da prevalência de certas doenças crónicas. Este aumento, que se tem verificado nos últimos anos, está estritamente relacionado com alterações dos estilos de vida; nomeadamente o tabagismo, o alcoolismo, os maus hábitos alimentares e o sedentarismo. O sedentarismo, característico das sociedades contemporâneas, é um factor de risco importante para uma grande parte das doenças crónicas não transmissíveis, nomeadamente para as doenças cardiovasculares. ${ }^{1-4}$

No nosso país, a prática de exercício físico de forma contínua e programada ao longo da vida é realizada por um escasso número de indivíduos. O eurobarómetro

*Interna do $1 .^{\circ}$ ano da Formação Específica de Medicina Geral e Familiar. Unidade de Saúde Familiar Horizonte - Unidade Local de Saúde de Matosinhos.
72.3, publicado em 2010 , indica que $55 \%$ da população portuguesa é inactiva. ${ }^{5}$ A prevalência de sedentarismo em Portugal é superior à da hipertensão arterial $(42 \%),{ }^{6}$ do tabagismo $(22 \%),{ }^{7}$ da obesidade $(15 \%)^{8} \mathrm{e}$ da diabetes mellitus (7.3\%). ${ }^{9} \mathrm{O}$ sedentarismo deveria ser considerado um problema de saúde pública pelas suas proporções endémicas. Neste sentido, é de extrema importância que ocorram alterações na nossa sociedade, nomeadamente, nas atitudes, nos valores e na conduta no que concerne à relação entre a saúde e o exercício físico.

A promoção de exercício físico representa assim, uma prioridade ao nível da saúde pública, sendo necessário intervir em várias frentes: individual, familiar, cuidados de saúde, comunitária e governamental. ${ }^{10} \mathrm{Ao}$ nível dos cuidados de saúde, os médicos de família desempenham um papel importante na promoção da 
saúde e na prevenção da doença, e portanto têm nas suas mãos a possibilidade de dar o seu contributo na resolução deste problema tão actual-a inactividade física.

É urgente abordar a prescrição de exercício físico como mais um acto médico, como se de um medicamento se tratasse. A prescrição de exercício físico deve ser vista como um processo pelo qual se recomenda um programa de exercícios (com uma correcta indicação da quantidade e da qualidade de exercício físico) de forma sistemática e individualizada, segundo as necessidades e preferências de cada um, com a finalidade de obter os maiores benefícios com os menores riscos. ${ }^{2,11}$

\section{OS BENEFÍCIOS DO EXERCÍCIO FÍSICO SÃO INDISCUTÍVEIS}

O exercício físico implica a existência da planificação de uma actividade, com o objectivo de melhorar a qualidade da saúde de cada indivíduo. ${ }^{2,11}$

Os benefícios do exercício físico são indiscutíveis, sendo este responsável pela: promoção do bem-estar psicológico e redução do stress, da ansiedade e dos sintomas depressivos, melhoria das funções cognitivas, melhoria da auto-imagem e da auto-estima, diminuição do absentismo laboral, ajuda no controlo da tensão arterial, do perfil lipídico e das glicemias, melhoria do padrão de sono, ajuda no controlo do peso, maior mineralização dos ossos em idades jovens, contribuindo para a prevenção da osteoporose e de fracturas em idades mais avançadas, prevenção e controlo das doenças crónicas. ${ }^{2-4,10-13}$

De acordo com os documentos orientadores da Organização Mundial da Saúde, a meta recomendada pela União Europeia e os seus Estados-membros para adultos saudáveis, com idade entre os 18 e os 65 anos, é de 30 minutos de exercício físico de intensidade moderada, 5 dias por semana; ou pelo menos 20 minutos de exercício físico de intensidade vigorosa, 3 dias por semana. A dose necessária de exercício físico pode ser acumulada em sessões de pelo menos 10 minutos e poderá compreender uma combinação de períodos de intensidades moderada e vigorosa. Para adultos com mais de 65 anos, deverão em princípio ser alcançadas metas idênticas às de adultos mais jovens. Nesta faixa etária são ainda especialmente importantes exercícios de flexibilidade, de fortalecimento muscular e de equilíbrio.
Os jovens em idade escolar devem participar diariamente em 60 minutos, ou mais, de actividades de intensidade moderada a vigorosa, sob formas adequadas do ponto de vista do crescimento, divertidas e que envolvam uma variedade de actividades. O tempo total poderá ser acumulado em sessões de pelo menos $10 \mathrm{mi}$ nutos. $^{14}$

Paralelamente à utilização da roda dos alimentos como instrumento para ajudar as pessoas a elaborarem um plano alimentar, a pirâmide de exercício físico ${ }^{15}(\mathrm{Fi}-$ gura 1) também pode ser usada pelos profissionais de saúde para o aconselhamento de exercício físico.

\section{OS MÉDICOS DE FAMÍLIA COMO PRESCRITORES DE EXERCÍCIO FÍSICO}

Na última década, vários estudos demonstraram que $o$ aconselhamento sobre exercício físico realizado pelos profissionais de saúde é eficaz..$^{10,16-22} \mathrm{~A}$ inclusão de exercício físico na prescrição médica provou ser um caminho viável para diminuir os níveis de sedentarismo da população em geral. ${ }^{19}$ No entanto, este papel exercido pelos profissionais de saúde necessita de um melhor reconhecimento. ${ }^{14}$

Um dos temas abordados na última reunião anual do American College of Sports Medicine, que teve lugar em Denver em Junho de 2011, foi precisamente o aconselhamento sobre exercício físico. O autor desta comunicação referiu que existe evidência que cerca de dois terços das pessoas estariam dispostas a serem mais fisicamente activas se os seus médicos realizassem um aconselhamento nesse sentido. Além disso, foi também referido que a maioria das pessoas considera que, se o aconselhamento sobre exercício físico fosse realizado por um médico activo e saudável, seria mais credível e motivador. Foi também salientado a necessidade dos actuais e futuros médicos compreenderem a importância da transmissão de orientações acerca do exercício físico a cada pessoa. ${ }^{23}$ Nesta mesma comunicação foram transmitidos os resultados de um estudo que revelou que os estudantes de medicina com hábitos saudáveis, que praticam exercício físico de forma regular, estão mais propensos a prescreverem exercício físico na sua prática clínica futura. ${ }^{23}$

Os médicos de família encontram-se numa posição privilegiada, não só pelo acompanhamento longitudinal e continuado da população em geral mas também 


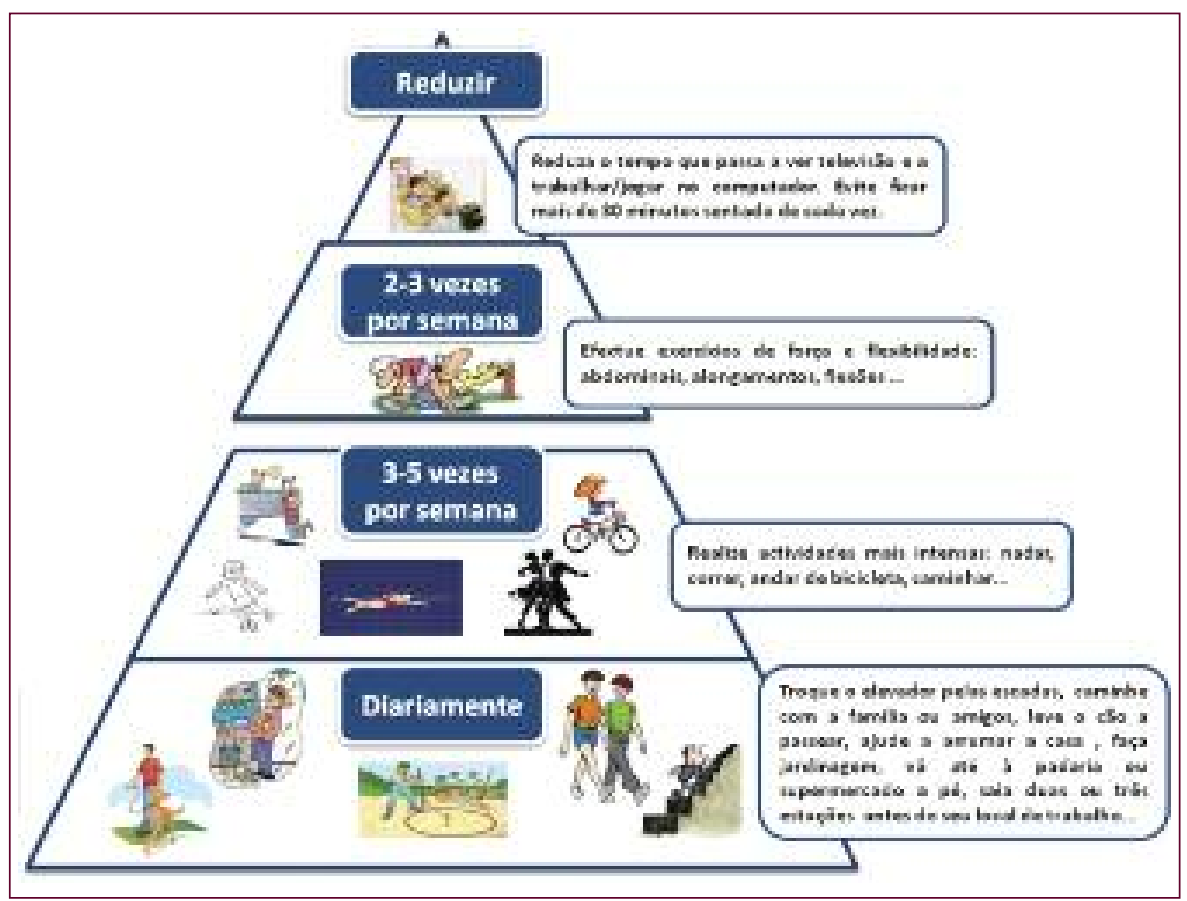

Figura 1. Pirâmide de exercício físico. Adaptação referência bibliográfica n. ${ }^{15}$. mentação, onde os médicos de família mostram maior actividade. ${ }^{26}$ As barreiras à prescrição de exercício físico, que podem explicar as diferenças observadas na prática de aconselhamento de mudança de comportamentos em saúde, incluem: a falta de tempo, a falta de formação e de qualificação, a inexistência de materiais/protocolos de aconselhamento e a falta de confiança na alteração de comportamentos. ${ }^{3,14,26-28}$ Para aumentar ainda mais este dilema, os doentes frequentemente identificam os seus médicos de família como fonte preferencial de aconselhamento de exercício físico. ${ }^{29,30}$ Apesar da maioria dos médicos de família considerarem o aconselhamento de exercício físico uma tarepelo conhecimento do meio familiar, ambiental e comunitário que rodeia os indivíduos. Os ensaios clínicos suportam o valor potencial do aconselhamento de exercício físico nos cuidados de saúde primários. Estes estudos revelaram que o nível de exercício físico dos indivíduos pode aumentar (pelo menos a curto prazo) através de um aconselhamento breve, focado e orientado por objectivos, moldado às necessidades de saúde/escolhas da pessoa e oferecido em múltiplos contactos (consultas de seguimento, contacto telefónico, via e-mail, sessões de grupo). ${ }^{22,24} \mathrm{O}$ aconselhamento de exercício físico deve conter um plano escrito com os objectivos a alcançar. ${ }^{25}$

Os dados disponíveis sugerem que os médicos de família revelam atitudes positivas relativamente às medidas preventivas em geral e que acreditam que o exercício físico é um determinante importante na prevenção. Surpreendentemente, a maioria dos médicos, nos cuidados de saúde primários, não regista os hábitos de exercício físico dos seus utentes e realiza um aconselhamento sobre exercício físico poucas vezes estruturado. O que contrasta com o aconselhamento de outras alterações de estilos de vida, como o tabagismo e a ali- fa complicada, uma mensagem sucinta nesta área pode ser um potente catalisador para motivar a mudança de comportamento. $^{24,26}$

Ajudar as pessoas a modificarem os seus comportamentos em saúde é uma tarefa árdua mas não impossível. O comportamento individual é determinado por múltiplos factores pessoais, institucionais, ambientais que operam e interagem a nível individual, interpessoal e comunitário. ${ }^{16} \mathrm{O}$ movimento é uma forma de comportamento que implica o indivíduo no seu todo, nas suas dimensões biológica, psicológica e social. ${ }^{30} \mathrm{O}$ aconselhamento individualizado implica uma avaliação dos níveis de exercício físico, dos níveis motivacionais e das preferências, bem como dos riscos de saúde relacionados com o exercício físico e o acompanhamento da evolução verificada. ${ }^{14}$

\section{MODELOS E TEORIAS DO COMPORTAMENTO}

Uma das dificuldades, mencionadas pelos profissionais de saúde, para modificar os estilos de vida dos indivíduos, é a falta de conhecimento das técnicas, modelos e teorias que são utilizadas na mudança de comportamentos em saúde. ${ }^{19,32}$ Entre os modelos e as teorias vi- 
sando a prática de exercício físico mais utilizados destacam-se: a teoria social cognitiva e o modelo transteórico. ${ }^{19}$

A teoria social cognitiva propõe a existência de múltiplas influências no comportamento humano e enfatiza que as alterações estão relacionadas com factores sociais e cognitivos. Um dos objectivos desta teoria é aumentar a auto-eficácia do indivíduo, que faz parte do controlo cognitivo, e o seu objectivo é aumentar a autoconfiança até níveis suficientes para iniciar e manter mudanças comportamentais específicas. Existem imensas técnicas baseadas nesta teoria, entre as quais auto-monitorização, definição de objectivos, auto-esforço, análise dos prós e contras da mudança de determinado comportamento, prevenção de recaídas e suporte social. ${ }^{33}$

O modelo transteórico de Prochaska e Diclemente avalia a fase de motivação em que a pessoa se encontra, determinada pela intenção em manter ou alterar um traço comportamental específico. Representa um avanço teórico fundamental na compreensão de quando, como e porquê as pessoas mudam os seus comportamentos relacionados com a saúde. O pressuposto básico deste sucesso reside no facto de considerar a mudança comportamental como um processo e não um acontecimento, e de que os indivíduos têm diferentes níveis de motivação ou disposição para a mudança. Assim, pessoas em diferentes fases do processo de mudança podem e devem beneficiar de intervenções distintas e diferenciadas, mais adequadas à fase em que se encontram no momento. ${ }^{34}$ Este modelo foi desenvolvido para avaliar os hábitos tabágicos e foi posteriormente adaptado para outros padrões de estilo de vida, como o exercício físico. ${ }^{35}$ Deste modo, o conceito central do modelo transteórico é a dimensão temporal representada pelas cinco fases do ciclo de mudança, pelas quais as pessoas passam na modificação de comportamentos em saúde (Quadro I). Apresenta-se como um modelo circular e não linear, uma vez que as pessoas podem evoluir ao longo das fases, assim como sair em qualquer ponto do processo, e por diversas vezes. ${ }^{34}$ Por isso, em cada fase motivacional existem estratégias (Quadro I) para ajudar o indivíduo a manter o seu nível de motivação.

\section{AS BARREIRAS DA PRÁTICA DE EXERCÍCIO FÍSICO}

Um dos aspectos mais importantes no aconselhamento de exercício físico é a discussão de estratégias para ultrapassar as barreiras mencionadas pelo indivíduo para alcançar a próxima fase no ciclo de motivação. As barreiras para a prática de exercício físico devem ser categorizadas como demográficas, socioculturais ou ambientais ou podem estar relacionadas com o próprio exercício físico. Tem sido demonstrado que a idade está inversamente correlacionada com os níveis de exercício físico e que os homens tendem a ser mais activos que as mulheres. Também há evidência que a percepção das barreiras varia de acordo com a idade, a classe social e o nível socioeconómico. ${ }^{19}$

Apesar de conscientes relativamente às consequências dos seus hábitos sedentários, as pessoas enumeram algumas razões para justificarem o seu comportamento: falta de tempo («levanto-me cedo e chego a casa tarde e cansado»), custos elevados (vestuário e calçado adequados), medo de lesões, reduzida auto-confiança, falta de um local adequado perto da sua residência. ${ }^{36}$

\section{A PRESCRIÇÃO DE EXERCÍCIO FÍSICO COMO MAIS UM ACTO MÉDICO}

A prescrição de exercício físico necessita de um conjunto de conhecimentos científicos e de normas elementares, fundamentais para que se obtenha um bom resultado. Quando se pretende realizar uma prescrição a este nível, deve-se esclarecer as suas indicações/objectivos para se evitarem más orientações, que podem induzir consequências nefastas para a saúde das pessoas. Não basta indicar que se deve fazer exercício, é necessário especificar os aspectos quantitativos e qualitativos do exercício físico, como se faz com qualquer outra medicação. Considera-se, mesmo, uma verdadeira prescrição, semelhante a outras terapêuticas, com as suas indicações e efeitos secundários. ${ }^{31}$

Portanto, o acto da prescrição de exercício físico deve conter os seguintes itens: tipo, frequência, duração, intensidade, regularidade, progressão e personalização. ${ }^{11,15,31}$ Contrariando, assim, o que se faz habitualmente na prática clínica, onde apenas se aborda o tema de uma forma leve, realizando-se um aconselhamento mínimo sem regras..$^{11,15,31}$

O tipo de exercício físico, em qualquer idade e com qualquer problema de saúde, deve, sempre que não haja contra-indicações, ter em consideração a motivação e as preferências dos indivíduos e ter objectivos bem delineados. Quando a pessoa a quem foi prescri- 


\begin{tabular}{|c|c|c|}
\hline Fase & Característica & Estratégias \\
\hline Pré-contemplação & $\begin{array}{l}\text { O indivíduo não pratica qualquer } \\
\text { exercício físico e não tem } \\
\text { intenção em fazê-lo }\end{array}$ & $\begin{array}{l}\text { - Pedir permissão para abordar os hábitos sedentários do indivíduo } \\
\text { - Inquirir o indivíduo sobre o que pensa dos seus hábitos sedentários } \\
\text { - Inquirir o indivíduo sobre o que sabe acerca dos riscos dos hábitos } \\
\text { sedentários e transmitir apenas a informação que ele deseja } \\
\text { - Expressar preocupação } \\
\text { - Pedir ao indivíduo para pensar ou ler sobre a prática de exercício } \\
\text { físico entre as consultas }\end{array}$ \\
\hline Contemplação & $\begin{array}{l}\text { O indivíduo não pratica } \\
\text { exercício físico, mas considera } \\
\text { fazê-lo }\end{array}$ & $\begin{array}{l}\text { - Ajudar a identificar as vantagens e as desvantagens da prática de } \\
\text { exercício físico } \\
\text { - Assinalar gentilmente as discrepâncias } \\
\text { - Identificar as barreiras para a prática de exercício físico e ajudar a } \\
\text { encontrar soluções } \\
\text { - Aumentar a referência das vantagens da prática de exercício físico, } \\
\text { enfatizando os benefícios para a saúde que o indivíduo identifica }\end{array}$ \\
\hline Preparação & $\begin{array}{l}\text { O indivíduo considera } \\
\text { envolver-se na prática de } \\
\text { exercício físico ou já está } \\
\text { envolvido mas não nos níveis } \\
\text { recomendados }\end{array}$ & $\begin{array}{l}\text { - Diminuir a referência das desvantagens da mudança } \\
\text { - Ajudar o indivíduo a ultrapassar barreiras } \\
\text { - Negociar com o indivíduo um plano que se adapte às suas actividades } \\
\text { - Niárias } \\
\text { - Regociar uma data para iniciar alguns ou todos os objectivos definidos } \\
\text { - Planear consultas de seguimento }\end{array}$ \\
\hline Acção & $\begin{array}{l}\text { O indivíduo já está envolvido } \\
\text { num exercício físico que está } \\
\text { dentro dos parâmetros } \\
\text { recomendados, mas por um } \\
\text { período inferior a } 6 \text { meses }\end{array}$ & $\begin{array}{l}\text { - Dar suporte e enfatizar os prós da mudança } \\
\text { - Ajudar a definir estratégias para superar uma recaída } \\
\text { - Ajudar a alterar o plano de acção se for necessário } \\
\text { - Discutir recursos disponíveis } \\
\text { - Planear consultas de seguimento }\end{array}$ \\
\hline Manutenção & $\begin{array}{l}\text { O indivíduo está envolvido num } \\
\text { exercício físico nos níveis } \\
\text { recomendados e tem mantido } \\
\text { esses níveis por um período } \\
\text { superior a } 6 \text { meses }\end{array}$ & $\begin{array}{l}\text { - Dar suporte e demonstrar admiração } \\
\text { - Reforçar atitudes positivas } \\
\text { - Inquirir sobre sentimentos e expectativas } \\
\text { - Ajudar a definir estratégias para superar uma recaída } \\
\text { - Planear consultas de seguimento }\end{array}$ \\
\hline Recaída & $\begin{array}{l}\text { O indivíduo volta a uma fase } \\
\text { anterior }\end{array}$ & $\begin{array}{l}\text { - Ajudar a ver a recaída como uma oportunidade de aprendizagem e de } \\
\text { preparação para a fase de acção seguinte } \\
\text { - Evidenciar que a recaída é uma experiência comum no processo de } \\
\text { mudança } \\
\text { - Rever todo o processo e apoiar }\end{array}$ \\
\hline
\end{tabular}

to exercício físico realiza uma actividade que lhe dá prazer, há um maior empenho da sua parte, o que influencia positivamente a adesão ao plano de exercício físico proposto. ${ }^{11,15,31}$ De salientar que o exercício aeróbio, fundamental em qualquer idade, é o tipo de exercício mais importante para manter e melhorar a resis- tência cardio-respiratória. ${ }^{31}$

O número de dias por semana nos quais se deve realizar o exercício físico negociado não é indiferente. A frequência está intimamente relacionada com a intensidade e com a duração, portanto a indicação do número de vezes por semana que se deve praticar exercício 
físico deverá ter em consideração estes dois factores. A frequência das sessões depende ainda da idade, dos problemas de saúde, da motivação e da disponibilidade individual de cada indivíduo. ${ }^{11,15,31}$

A intensidade é provavelmente o parâmetro mais importante mas também o mais complicado. Quando se decide a intensidade do exercício físico, deve ter-se em consideração os níveis mais adequados a cada pessoa, para que a actividade seja realizada com segurança. Para definir-se a intensidade usam-se alguns métodos, dos quais os mais comuns são: determinação da frequência cardíaca máxima, critérios subjectivos (o indivíduo deve ser capaz de conversar durante o exercício físico e ter a sensação permanente de estar preparado para prolongar o exercício físico sem esforço) e determinação da capacidade funcional máxima. ${ }^{11,15,31}$ Relativamente à determinação da frequência cardíaca máxima, esta pode ser obtida de uma forma simples para o indivíduo, subtraindo a sua idade a duzentos e vinte. As pessoas com uma capacidade funcional muito baixa, como os idosos, os sedentários, os obesos e os doentes cardíacos devem iniciar um exercício físico com uma intensidade de $40 \%$ da frequência cardíaca máxima, fazendo depois uma progressão lenta até atingir $60-70 \% \cdot{ }^{31} \mathrm{~A}$ unidade de determinação da capacidade funcional máxima é o MET, que expressa a intensidade do metabolismo em repouso num dado momento. Assim, um MET corresponde ao metabolismo em repouso, dois MET ao dobro do metabolismo em repouso e assim sucessivamente. Existem tabelas onde estão expressas em MET as intensidades das actividades mais correntes. ${ }^{15,31}$

Qualquer exercício físico deve ser regular, portanto deve-se praticar um exercício físico de forma contínua e sistemática. Qualquer programa de exercício físico deve permitir uma progressão lenta, com um aumento gradual da duração, da frequência e da intensidade. Cada sessão deve iniciar-se por um período de aquecimento proporcional à sua duração e terminar com um período de arrefecimento. ${ }^{11,15,31}$

A personalização do exercício físico engloba vários itens: sexo, idade, passado desportivo, gostos, tempo livre, horários preferenciais, grau de solicitação energética da actividade profissional e doméstica, objectivos e motivação de cada um, antecedentes pessoais, os problemas médicos actuais e a medicação habitual. Deve, ainda, ter-se em consideração as capacidades específicas de cada pessoa (resistência aeróbia, resistência anaeróbia, velocidade, força, coordenação neuromuscular e flexibilidade)..$^{11,31}$

$\mathrm{O}$ respeito por estes princípios contribui para a individualização e personalização do exercício físico. Aplicam-se aos indivíduos de todas as idades e capacidades funcionais, independentemente da presença ou não de factores de risco e eventuais doenças. ${ }^{31}$

Ser um mero agente de saúde que dá autorização e ordens no que concerne à prática de exercício físico certamente não trará qualquer benefício para a pessoa. Ser conselheiro, avaliar e ajudar o indivíduo negociando o plano de exercício físico com o próprio, é sem dúvida a melhor solução. ${ }^{19}$ No Quadro II apresentam-se alguns conselhos para o aconselhamento de exercício físico numa primeira abordagem e em consultas posteriores. ${ }^{19,24}$

A consulta programada de um médico de família é abrangente e por vezes o tempo é escasso para abordar vários assuntos numa só consulta. Contudo, o facto dos médicos de família terem o privilégio de fazer um acompanhamento longitudinal dos seus utentes permite repartir os vários assuntos a tratar pelas diversas consultas e definir objectivos para cada uma delas. Podendo assim incluir também o aconselhamento de exercício físico numa consulta definida.

\section{PROGRAMAS BASEADOS NO ACONSELHAMENTO DE EXERCÍCIO FÍSICO}

Nos últimos anos, os médicos têm vindo a aceitar gradualmente o aconselhamento de exercício físico como uma estratégia preventiva. Muitos países têm desenvolvido programas que se baseiam no aconselhamento de exercício físico durante o tratamento médico.

O projecto physician-based assessment and counseling for exercise (PACE) é um programa de aconselhamento de exercício físico baseado em sessões médicas nos cuidados de saúde primários, no qual a orientação começa pela identificação da fase motivacional. As orientações do programa sugerem três ou cinco minutos de aconselhamento por visita médica, durante a qual tópicos relevantes para o indivíduo são discutidos e objectivos para o exercício físico são estabelecidos. $\mathrm{O}$ programa engloba três estádios: «getting out of the 


\begin{tabular}{|c|c|}
\hline Primeira abordagem & Consultas posteriores \\
\hline - Avaliar o nível de exercício físico & $\begin{array}{l}\text { - Reavaliar o nível de exercício físico e conferir o tipo de exercício físico que está a } \\
\text { ser realizada }\end{array}$ \\
\hline $\begin{array}{l}\text { - Identificar a fase motivacional em que o } \\
\text { indivíduo se encontra e os benefícios da } \\
\text { prática de exercício físico }\end{array}$ & $\begin{array}{l}\text { - Rever a fase motivacional e se os benefícios identificados pelo indivíduo na } \\
\text { primeira consulta se mantêm }\end{array}$ \\
\hline $\begin{array}{l}\text { - Estabelecer a melhor abordagem de } \\
\text { acordo com a fase motivacional }\end{array}$ & $\begin{array}{l}\text { - Reavaliar as barreiras e perceber a razão porque os conselhos transmitidos na } \\
\text { primeira sessão para as ultrapassar não foram seguidos }\end{array}$ \\
\hline $\begin{array}{l}\text { - Envolver o indivíduo na identificação } \\
\text { dos seus próprios objectivos e da } \\
\text { escolha do tipo de exercício físico }\end{array}$ & - Conferir se existe necessidade de alterar a proposta \\
\hline $\begin{array}{l}\text { - Identificar barreiras à prática de } \\
\text { exercício físico e ajudar na identificação } \\
\text { de soluções para as ultrapassar }\end{array}$ & $\begin{array}{l}\text { - Identificar as alterações que ocorreram como consequência do exercício físico. } \\
\text { Reforçar as mudanças que foram obtidas. }\end{array}$ \\
\hline $\begin{array}{l}\text { - Conduzir o indivíduo à repetição da } \\
\text { proposta estabelecida como um } \\
\text { compromisso }\end{array}$ & $\begin{array}{l}\text { - Ter a certeza que o doente entendeu a razão de algumas barreiras não terem sido } \\
\text { ultrapassadas }\end{array}$ \\
\hline $\begin{array}{l}\text { - Registar todas a orientações do plano } \\
\text { de uma forma objectiva e clara }\end{array}$ & - Identificar a necessidade de rever os objectivos e tipo de exercício físico \\
\hline $\begin{array}{l}\text { - Rever todos os benefícios que o } \\
\text { indivíduo obterá }\end{array}$ & $\begin{array}{l}\text { - Levar o indivíduo a repetir o programa estabelecido por ambos, e tornar claro que } \\
\text { este programa é um compromisso entre os dois, mas principalmente que é } \\
\text { realizado para o indivíduo }\end{array}$ \\
\hline $\begin{array}{l}\text { - Ter a certeza que o indivíduo entendeu } \\
\text { tudo o que foi explicado e negociado }\end{array}$ & - Reescrever todas as orientações de uma forma objectiva e clara \\
\hline
\end{tabular}

chair» (pré-contemplativo), "planning the first steps» (contemplativo) e «keeping the PACE» (activo). Adicionalmente, os médicos avaliam as fontes do suporte social, identificam as possíveis barreiras para a prática de exercício físico e ajudam as pessoas a encontrar caminhos para ultrapassar essas barreiras, demonstrando assim a confiança de que o indivíduo manterá a adesão ao programa de exercício físico estabelecido. ${ }^{19,36}$

O projecto physically active for life (PAL) foi desenvolvido com o intuito de reduzir os hábitos sedentários entre os idosos. Como o projecto PACE, o modelo PAL foi desenvolvido nos cuidados de saúde primários e utiliza o modelo transteórico da mudança de comportamentos, tendo como objectivo ultrapassar as barreiras para a prática de exercício físico. O modelo PAL integra características cognitivas, instrumentais, comportamentais e sociais; numa abordagem centrada na educação do indivíduo. Isto é realizado usando uma série de questões e demonstrações baseadas nos $5 \mathrm{~A}$ s (abordar, aconselhar, avaliar, ajudar e acompanhar). ${ }^{19,36}$

O projecto the step test exercise prescription (STEP) é outro programa de aconselhamento sobre exercício físico realizado nos cuidados de saúde primários, através do qual os médicos de família fornecem aconselhamento sobre exercício físico tendo em conta orientações publicadas, entregam um papel com a descrição dos benefícios do exercício físico e prescrevem exercício físico tendo em conta a capacidade aeróbia e a frequência cardíaca de cada indivíduo. ${ }^{26,36}$

No projecto increasing and maintaining physical activity by connecting and tracking participants (IMPACT) é realizado um contacto telefónico ou um contacto via e-mail de forma periódica, através do qual os profissionais de saúde esclarecem dúvidas, sugerem formas de ultrapassar as barreiras e enviam informações adicionais às transmitidas em consulta. ${ }^{36}$ 
No projecto green prescription as orientações e a prescrição de exercício físico são efectuadas de acordo com o grupo etário, problemas de saúde, capacidade e actividades diárias. Inicialmente são transmitidas verbalmente e posteriormente são escritas num papel para fornecer ao indivíduo. ${ }^{22,36}$

\section{SERÁ QUE ESTAMOS PREPARADOS?}

O programa nacional de intervenção integrada sobre determinantes da saúde relacionados com estilos de vida reitera o exercício físico como um dos principais factores determinantes a considerar, a par da alimentação, tabaco, álcool e gestão de stress. ${ }^{4}$ Contudo, actualmente, no nosso país não existe qualquer projecto de aconselhamento de exercício físico implementado nos cuidados de saúde primários à semelhança do que se verifica noutros países. Cada médico de família, perante as condições existentes, realiza um aconselhamento de exercício físico baseado nos conhecimentos e experiência que tem. Estes profissionais não possuem actualmente protocolos, instrumentos, ferramentas de aconselhamento estandardizados que permitam um aconselhamento eficaz.

Será que na actual sociedade, com hábitos sedentários tão implementados e com o fardo de um aumento da prevalência de algumas doenças resultantes da inactividade física, não se justificaria um projecto com uma abordagem semelhante à realizada noutros países? Será que não seria útil a criação de um local para o registo dos hábitos de exercício físico nos sistemas informáticos como já existe para o álcool e o tabagismo? Será que os médicos de família estão sensibilizados para este tipo de aconselhamento, que engloba duas vertentes importantíssimas da sua prática clínica: a promoção da saúde e a prevenção da doença?

É urgente rever o aconselhamento de exercício físico nos cuidados de saúde primários. É fundamental ver a prescrição de exercício físico como mais um acto médico. Por um lado, os médicos de família precisam rever o seu papel na promoção de exercício físico, por outro lado as faculdades de medicina necessitam de realizar uma revisão crítica aos seus currículos, assegurando a preparação dos seus estudantes e futuros clínicos para esta abordagem. ${ }^{24,37}$

A prescrição de exercício físico deve tornar-se uma componente integral da prática clínica nos cuidados de saúde primários. É difícil imaginar uma abordagem com maior potencial na promoção da saúde da nossa população. ${ }^{38}$

\section{CONCLUSÃO}

Estamos perante uma sociedade com hábitos sedentários e portanto o risco do aumento da prevalência de doenças relacionadas com o sedentarismo é elevadíssimo. Sabemos que o exercício físico é importante para a saúde e bem-estar da população em geral, contudo muitas vezes o tempo escasseia para abordar diversos temas numa só consulta e não temos uma preparação técnico-científica adequada.

A necessidade de mudança de comportamentos em relação ao exercício físico é dupla. Os indivíduos devem realizar uma revisão às prioridades na sua vida e os médicos devem rever o seu papel na promoção da saúde e na prevenção da doença.

Está demonstrada a eficácia do aconselhamento de exercício físico baseado nos modelos de mudança de comportamentos e nos instrumentos/protocolos de apoio. Existem estratégias definidas para a realização de aconselhamento de exercício físico durante as consultas em cuidados de saúde primários.

Presentemente prescrever exercício físico é «imperativo ético», tão importante como a prescrição de um plano alimentar ou de um fármaco.

Existem condições para que o aconselhamento de exercício físico não seja negligenciado. Não vamos desistir dos nossos utentes, vamos ajudá-los a tornaremse mais activos. Mexam-se pela saúde da nossa sociedade!

Está lançado o desafio para todos nós!

\section{REFERÊNCIAS BIBLIOGRÁFICAS}

1. Proper KI, singh AS, van Mechelen W, Chinapaw MJ. Sedentary behaviors and health outcomes among adults: a systematic review of prospective studies. Am J Prev Med 2011 Feb; 40 (2): 174-82.

2. Teixeira PJ, Sardinha LB, Barata JL, edcitores. Nutrição, exercício e saúde. Lisboa: Lidel; 2008. p. 181-91.

3. Weidinger KA, Lovegreen SL, Elliott MB, Hagood L, Haire-Joshu D, mcGill $\mathrm{JB}$, et al. How to make exercise counseling more effective: lessons from rural America. J Fam Pract 2008 Jun; 57 (6): 394-402.

4. Direcção Geral de Saúde. Actividade física e desporto: actuação ao nível da educação para a saúde. Circular informativa. Lisboa: Ministério da Saúde; 2007.

5. European Comission. Sport and physical activity. Eurobarometer 72.3 . Disponível em: http://ec.europa.eu/public_opinion/archives/ebs/ebs_ 
334_en.pdf [acedido em 222/04/2011].

6. Macedo ME, Lima MJ, Silva AO, Alcântara P, Ramalhinho V, Carmona J. Prevalência, conhecimento, tratamento e controlo da hipertensão em Portugal. Estudo PAP. Rev Port Cardiol 2007 Jan; 26 (1): 21-39.

7. Bridgehead International, EQUIPP: Europe Quitting: Progress and Pathways, London, 2011. Disponível em: http://www.ersnet.org/images/stories/weekly/EQUIP_REPORT_COMPLETE.PDF [acedido em 22/04/2011].

8. Direcção Geral de Saúde. Plataforma contra a obesidade. Disponível em: http://www.plataformacontraaobesidade.dgs.pt [acedido em 22/04/2011].

9. Ministério da Saúde. Diabetes: factos e números 2010. Relatório Anual do Observatório Nacional da Diabetes. Disponível em: http://www. rncci.min-saude.pt/SiteCollectionDocuments/RelatorioAnualDiabetes_2010.pdf [acedido em 22/04/2011].

10. Grandes G, Sanchez A, Sanchez-Pinilla RO, Montoya I, Lizarraga K, Serra J; PEPAF Group. Effectiveness of physical activity advice and prescription by physicians in routine primary care: a cluster randomized trial. Arch Intern Med 2009 Apr 13; 169 (7): 649-701.

11. Institut Catalá de la Salut. Manual d activitat física en atenció primària. Barcelona: Institut Català de la Salut; 2009.

12. Warburton DE, Nicol CW, Bredin SS. Prescribing exercise as preventive therapy. CMAJ 2006 Mar 28; 174 (7): 961-74.

13. Villanueva T, Khan KM. Exercise for good health. Student BMJ 2004 Jul; 12: 266-7.

14. Instituto do Desporto de Portugal. Orientações da União Europeia para a actividade física: Políticas recomendadas para a promoção da saúde e do bem-estar. Disponível em: http://www.idesporto.pt/ficheiros/File/ Livro_IDPfinalJan09.pdf [acedido em 22/04/2011].

15. American College of Sports Medicine. ACSM's Guidelines for exercise testing and prescription. Phuladelphia, PA: Lippincott Williams \& Wilkins; 2009.

16. Grandes G, Sanchez A, Cortada JM, Balague L, Calderon C, Arrazola A, et al. Is integration of healthy lifestyle promotion into primary care feasible? Discussion and consensus sessions between clinicians and researchers. BMC Health Serv Res 2008 Oct 14; 8: 213.

17. Petrella RJ, Lattanzio CN. Does counseling help patients get active? Can Fam Physician 2002 Jan; 48:72-80.

18. Smith BJ, Bauman AE, Bull FC, Booth ML, Harris MF. Promoting physical activity in general practice: a controlled trial of written advice and information materials. Br J Sports Med 2000 Aug; 34 (4): 262-7.

19. Ribeiro MA , Martins MA, Carvalho CR. The role of physician counseling in improving adherence to physical activity among the general population. Sao Paulo Med J 2007 Mar; 125 (2): 115-21.

20. Hellénius ML, Sundberg CJ. Physical activity as medicine: time to translate evidence into clinical practice. Br J Sports Med 2011 Mar; 45 (3): 158.

21. Sørensen JB, Skovgaard T, Puggaard L. Exercise on prescription in general practice: a systematic review. Scand J Prim Health Care 2006 Jun; 24 (2): 69-74.

22. Martin SN, Crownover BK, Kovach FE. What's the best way to motivate patients to exercise? J Fam Pract 2010 Jan; 59 (1): 43-4.

23. American College of Sports Medicine. Fit doctors more likely to encourage patients to exercise. Disponível em: http://www.acsm.org/
AM/Template.cfm?Section=ACSM_News_Releases\&TEMPLATE=/CM/ ContentDisplay.cfm\&CONTENTID=15938 [acedido em 30/06/2011].

24. Khan KM, Weiler R, Blair SN. Prescribing exercise in primary care. BM] 2011 Jul 15; 343: d4141. doi: 10.1136/bmj.d4141.

25. Carroll JK, Fiscella K, Epstein RM, Jen-Pierre P, Figueroa-Moseley C, Williams GC, et al. Getting patients to exercise more: a systematic review of underserved populations. J Fam Pract 2008 Mar; 57 (3): 1706.

26. Petrella RJ, Wight D. An office-based instrument for exercise counseling and prescription in primary care: the Step Test Exercise Prescription (STEP). Arch Fam Med 2000 Apr; 9 (4): 339-44.

27. Meriwheter RA, Lee JA, Lafleur AS, Wiseman P. Physical activity counseling. Am Fam Physician 2008 Apr 15; 77 (8): 1129-36.

28. Chakravarthy MV, Joyner MJ, Booth FW. An obligation for primary care physicians to prescribe physical activity to sedentary patients to reduce the risk of chronic health conditions. Mayo Clin Proc 2002 Feb; 77 (2): 165-73.

29. Van der Ploeg HP, Smith BJ, Stubbs T, Vita P, Holford R, Bauman AE. Physical activity promotion: are GPs getting the message? Aust Fam Physician 2007 Oct; 36 (10): 871-4.

30. Swedish Council on Technology Assessment in Health Care. Methods of Promoting Physical Activity: a systematic review. 2007. disponível em: http://www.sbu.se/upload/Publikationer/Content1/1/Fysisk_ sam_ENG.pdf [acedido em 21/04/2011].

31. Nunes L. A prescrição de actividade física. Lisboa: Caminho; 2009.

32. Whitlock EP, Orleans CT, Pender N, Allan J. Evaluating primary care behavioral counseling interventions: an evidence-based approach. Am J Prev Med 2002 May; 22 (4): 267-84.

33. Bandura A. Social foundations of thought and action: a social cognitive theory. Englewood Cliffs: Prentice Hall; 1986.

34. Sallis J, Owen N. Physical activity and behavioral medicine. Thousand Oaks: Sage Publications; 1999.

35. Marcus BH, Simkin LR. The transtheoretical model: applications to exercise behavior. Med Sci Sports Exerc 1994 Nov; 26 (11): 1400-4.

36. Sociedade Brasileira de Medicina de Família e Comunidade e Sociedade Brasileira de Medicina do Esporte. Rastreamento do sedentarismo em adultos e intervenções na promoção da atividade física na atenção primária à saúde. Projeto Diretrizes 2009. Disponível em: http://www. projetodiretrizes.org.br/8_volume/36-Rastreamento.pdf [acedido em 30/06/2011].

37. Connaughton AV, Weiler RM, Connaughton DP. Graduating medical students' exercise prescription competence as perceived by deans and directors of medical education in the United States: implications for Healthy People 2010. Public Health Rep 2001 May-Jun; 116 (3): 22634.

38. Eakin EG, Glasgow RE, Riley KM. Review of primary care-based physical activity intervention studies: effectiveness and implications for practice and future research. J Fam Pract 2000 Feb; 49 (2): 158-68.

\section{INFORMAÇÃO ADICIONAL}

MacAuley D, editor. Oxford Handbook of Sport and Exercise Medicine. Oxford, NY: Oxford University Press; 2007.

Heyward VH. Advanced Fitness Assessment and Exercise Prescription. Champaign, IL: Human Kinetics; 2010. 
Swedish National Institute of Public Health. Physical activity in the prevention and treatment of disease. Stockholm: SNIPH; 2010. Disponível em: http://www.fhi.se/PageFiles/10682/Physical-Activity-Prevention-Treatment-Disease-webb.pdf [acedido em 30/06/2011].

\section{CONFLITO DE INTERESSES}

O autor declara não existir conflitos de interesse na elaboração deste artigo.

\author{
ENDEREÇO PARA CORRESPONDÊNCIA \\ Diana Carneiro \\ Rua S. José, n. ${ }^{\circ} 140$ - Balasar \\ 4570-055 Póvoa de Varzim \\ E-mail: dianaaccarneiro@gmail.com
}

Recebido em 24/05/2011

Aceite para publicação em 07/08/2011

\section{ABSTRACT}

\section{THE PRESCRIPTION OF PHYSICAL EXERCISE: INCLUSION IN THE OFFICE VISIT}

The proportion of individuals in the population with sedentary habits has increased in the last few years. A sedentary lifestyle is an important risk factor for chronic non-communicable diseases, especially for cardiovascular diseases. Therefore it is important to promote changes in attitudes, values and practices related to health and exercise.

There is evidence that the inclusion of physical exercise in a medical prescription is one way to approach sedentary habits in the general population. Family physicians are in a privileged position to try to change behavior, not only because of their continuous contact with a population over time but also because their knowledge of the family, the environment and the community.

The prescription of exercise should be understood as a process in which an exercise program is recommended in a systematic and individualized way based on the needs and preferences of each person, in order to obtain benefits without increasing risks. Physicians should not just state that one should do exercise. As for medications, it is necessary to specify the quantitative and qualitative aspects of exercise.

The purpose of this article is to discuss the prescription of exercise and to propose it as one of the medical activities that can be performed in an office visit. The benefits of exercise, the role of the physician as a promoter of exercise, the models and theories explaining exercise behaviour, the characteristics of exercise prescriptions, the strategies for their implementation in clinical practice, and primary care programs for advice on exercise are discussed.

As the rates of sedentary habits in the population are increasing, it is important to promote exercise counseling in primary care.

Key-words: Sedentary Lifestyle; Behavior Change; Exercise; Counseling. 